Sir

\section{Locking the Luer lock}

I read with interest the article on 'Patient safety in cataract surgery'. ${ }^{1}$ As stated in the article, penetration of the globe during periocular injections is rightly, a reportable incident. Complications however also occur with the use of syringes intracamerally. I would like to draw your attention to the case of an 85-year-old gentleman who underwent uneventful left phacoemulsification surgery. At the end of the procedure, a Luer lock syringe was used with a 27-gauge Rycroft cannula for stromal hydration around the corneal section. The cannula tip dislodged under pressure and penetrated the eye through the wound to cause a hyphaema, choroidal haemorrhage, and total retinal detachment.

He was managed initially with a vitrectomy, removal of the intraocular lens with drainage of the suprachoroidal haemorrhage, and silicone oil internal tamponade. He developed proliferative vitreoretinopathy with a perisilicone retinal detachment that was managed with a retinectomy and silicone oil top-up procedure 6 weeks later. The silicone oil was successfully removed 3 months later with secondary anterior chamber intraocular lens implantation; his final best-corrected visual acuity was $6 / 18$.

Case reports of similar occurrences have been reported in the literature, one with the use of Luer locked syringe during hydrodissection ${ }^{2}$ and one with a nonthreaded syringe. $^{3}$
In both ours and the above-mentioned case, ocular penetration occurred despite the use of a Luer lock. Crossthreading of the cannula prevented it from locking into place and the force used to push fluid through the cannula caused it to 'shoot' into the eye. Following this adverse clinical event, our unit has adopted a protocol where cannulas on Luer lock syringes are checked and locked before use, by both scrub nurse and surgeon. No similar incidents have occurred in over 5000 subsequent cataract procedures.

\section{References}

1 Kelly SP, Astbury NJ. Patient safety in cataract surgery. Eye 2006; 20: 275-282.

2 Munshi V, Sampat V, Pagliarini S. J Cataract Refract Surg 2005; 31(2): 450-451.

3 Dinakaran S, Kayarkar VV. J Cataract Refract Surg 1999; 25(5): 720-721.

P Pandey and R Scott

Birmingham Midland Eye Centre, City Hospital, Dudley Road, Birmingham B18 7QH, UK

Correspondence: P Pandey,

Tel: + 447974961129 ;

Fax: + 441215076887 .

E-mail: pravin1@gmail.com

Eye (2007) 21, 449. doi:10.1038/sj.eye.6702624; publication online 13 October 2006 